\title{
Abdominal Rhabdomyosarcoma
}

National Cancer Institute

\section{Source}

National Cancer Institute. Abdominal Rhabdomyosarcoma. NCI Thesaurus. Code C151982.

Rhabdomyosarcoma involving the organs and structures in the abdomen. 